\title{
ESTRUCTURA Y COMPONENTE EMPÍRICO DE LA TEORÍA DEL INTERCAMBIO ECONÓMICO
}

\author{
Amparo GOMEz RODRIGUEZ \\ Dpto. de Historia y Filosofía de la Ciencia. \\ Universidad de la Laguna
}

RESUMEN. En este artículo se aborda la axiomatización informal de la teoría del intercambio. La idea motriz del análisis es que la reconstrucción permite la clarificación y precisión de la estructura de la teoría pero también de los problemas conceptuales y de aplicación que ésta presenta, permitiendo establecer qué partes de la teorla necesitan investigación ulterior. En el caso que se analiza se prestará especial atención al problema del componente empírico de la teoría del intercambio indagando qué elementos estructurales están implicados en las dificultades para disponer de un conjunto I de aplicaciones efectivas y de un rendimiento empírico adecuado.

Palabras Clave. Axiomatización, economía de intercambio, función de utilidad, maximización de la utilidad términos t-teóricos y t-no teóricos, constricciones, aplicaciones, modelos, contenido empírico.

ABSTRACT. In this article the author examines the informal axiomatization of exchange theory of neoclassical economics. The central issue of this analysis is that the structuralist axiomatic reconstruction makes possible the clarification and precision of theory's structure, but also of its conceptual problems and applications. In the case that we analyse we pay attention to the problem of empirical content of exchange theory, researching what structural elements are concerned with the difficul ties to have a set I of effective applications and, therefore, appropiate empirical outcomes. 
KEYWORDS. Axiomatization, exchange economics, utility function, maximization of utility, $t$-theoretical and $t$-no theoretical terms, constraints, applications, models, empirical content.

Las ventajas de disponer de teorías científicas axiomatizadas pueden sintetizarse básicamente en los siguientes aspectos: la axiomatización ofrece una descripción cuidadosa de la teoría, presenta un sumario de la investigación, permite examinar las consecuencias de sus axiomas y establecer qué partes de la teoría están sólidamente establecidas y qué partes no. En ciencias sociales ha existido una considerable dificultad para establecer axiomatizaciones efectivas de sus teorías con la excepción de algunas teorías de la economía.

En este artículo se examinará la axiomatización conjuntista de la teoría del intercambio de la economía neoclásica. Se tratará de establecer en qué medida esta axiomatización cumple el cometido de ofrecer una descripción de la teoría, con una presentación lo más precisa posible de su estructura y modelos $\mathrm{y}$, en qué medida, este objetivo presenta problemas y especificidades. La idea motriz de este análisis es que la reconstrucción estructural permite la clarificación y precisión de las teorías pero, también, de los problemas conceptuales y las aplicaciones que éstas presentan mostrando así qué partes de las teorías necesitan investigación ulterior. La axiomatización informal posibilita, por tanto, la discusión de importantes cuestiones conceptuales y epistemológicas de las teorías, de sus relaciones con otras teorías y con la realidad empírica. En el caso que se analiza se prestará especial atención al problema del componente empírico de la teoría del intercambio indagando qué elementos estructurales están implicados en las dificultades para disponer de un conjunto de aplicaciones efectivas y de un rendimiento empírico adecuado.

\section{La axiomatización conjuntista}

La axiomatización informal de las teorías científicas se basa en la definición de un predicado mediante nociones conjuntistas que explicita la estructura (formal-matemática) de la teoría ${ }^{1}$. Esta definición establece las condiciones (axio-

${ }^{1}$ La axiomatización informal proviene del trabajo de Boubarki (1968) y su extensión a las ciencias empíricas por P. Suppes, J. C. McKinsey y A. C. Sugar, 1953, J. Sneed, 1979 o 1987, Stegmuller,1970, 1975 o 1979, U. Moulines, 1975, 1982 o 1987, W. Balzer, 1982, 1985, o 1997. 
mas) que debe cumplir cualquier entidad para ser modelo de dicha estructura (se trata de que la extensión del predicado en cuestión sea la clase de los modelos de la teoría). Las teorías se identifican con clases de modelos que satisfacen en diversos grados la definición conjuntista del predicado ${ }^{2}$. Una teoría empírica queda determinada por los sistemas empíricos que son modelos de la teoría.

Los elementos centrales de una teoría son el conceptual o núcleo (K) (definición conjuntista de leyes, conceptos y relaciones) y el componente empírico, las aplicaciones propuestas (I). Una teoría $\mathrm{T}=\mathrm{K}, \mathrm{I}$.

El núcleo está constituido por las leyes y otros elementos present ados en forma axiomática, es decir, por la definición en lógica matemática de esos elementos y de las leyes de la teoría (en el caso de la mecánica clásica de partículas el segundo principio de Newton y el principio de acción y reacción).

Entre los elementos incluidos en el núcleo están los modelos que satisfacen en diversos grados los axiomas del predicado conjuntista. Estos son los modelos efectivos M, los modelos potenciales Mp y modelos potenciales parciales Mpp. Los modelos efectivos cumplen todos los axiomas del predicado incluyendo el axioma (o axiomas) que enuncia la ley (o leyes) de la teoría. Los modelos potenciales satisfacen todos los axiomas con la excepción de los que refieren a las leyes propiamente dichas y los potenciales parciales sólo satisfacen los axiomas que refieren a los elementos no teóricos. Esto supone distinguir los modelos que usan todo el aparato conceptual de la teoría de aquellos que sólo usan conceptos previamente disponibles.

Además en el núcleo están las restricciones que son las condiciones de ligadura, $\mathrm{CL}$, (relaciones entre modelos de la teoría) y las relaciones interteóricas, $\mathrm{RI}$, (relaciones entre teorías que forman una red). El núcleo $\mathrm{K}=<\mathrm{Mp}, \mathrm{Mpp}, \mathrm{M}$, $\mathrm{L}, \mathrm{RI}>$.

CL y RI son importantes ya que afectan al dominio I de aplicaciones propuestas de una teoría. El conjunto de aplicaciones de una teoría refiere a las par-

${ }^{2}$ Un ejemplo canónico de reconstrucción conjuntista de una teoría es la de la Mecánica Clásica de Partículas cuya exposición fue llevada a cabo por Sneed, McKinsey y Sugar, 1953. Ver la exposición de esa axiomatización en J. Mosterín 1984, pp.162- 164 y 179. o J. Echeverría, ] 999, pp. $178-184$. 
tes de la realidad a las que se ha aplicado efectivamente el núcleo conceptual $\mathrm{K}$. El conjunto de aplicaciones propuestas son un subconjunto del conjunto Mpp; I $\subseteq$ Mpp.

T es una teoría si existen Mp, Mpp, M, CL, RI e I tal que:

(1) $\mathrm{K}=<\mathrm{Mp}, \mathrm{Mpp}, \mathrm{M}, \mathrm{CL}, \mathrm{RI}>$ es un núcleo teórico.

(2) $\mathrm{I} \subseteq \mathrm{Mpp}$

(3) $\mathrm{T}=\langle\mathrm{K}, \mathrm{I}\rangle$.

Si ésta es la estructura sincrónica de una teoría su evolución diacrónica, o historia de la teoría, puede reconstruirse con las nociones de elemento teórico y red teórica.

\section{La Teoría del Intercambio}

Algunas teorías de la economía han sido axiomatizadas en términos conjuntistas. Este es el caso de la teoría del valor marxista, la teoría del valor, la teoría del equilibrio o la teoría del intercambio de la economía neoclásica ${ }^{3}$. Nos centraremos en la axiomarización de la teoría del intercambio económico (TIE), que según Balzer constituye el núcleo de la microeconomía, basándonos en la reconstrucción propuesta por este autor ${ }^{4}$. Al hilo de la misma plantearemos cierto tipo de cuestiones teóricas que afectan al caso examinado y a la reconstrucción conjuntista de otras teorías de la microeconomía.

Los elementos o términos primitivos de la teoría del intercambio económico son:

J Personas

G Clases de Bienes.

3 U. Moulines, 1982, y U. Moulines y I. Jane, 1981. A. García de la Sienra, 1980. E. Händler 1980, y W. Balzer, 1982, $1985,1997$.

4 W. Balzer, 1997, pp. 79-145 (original de 1982 y revisado en 1985). Balzer, 1982, pp. 2346. Balzer, 1985, pp. 1 85-200. Balzer refiere a "pure exchange economics", que es traducido en la versión española por" economía de trueque». Nosotros usamos el término «economía de intercambion. 


\section{q Cantidades de Bienes. \\ p Precios. \\ U Utilidad (beneficio) ${ }^{5}$.}

Balzer (1997:91) los define:

1) $\mathrm{J}=\left\{\pi_{1}, \ldots, \pi_{\mathrm{m}}\right\} \quad$ es un conjunto finito (de personas).

2) $G=\left\{\gamma_{i}, \ldots, \gamma_{n}\right\} \quad$ es un conjunto finito (de clases de bienes).

3) $\mathrm{q}: \mathrm{J} \times \mathrm{G} \rightarrow \mathrm{R}_{\mathrm{o}}^{+} \quad$ (distribución de bienes).

4) $\mathrm{p}: \mathrm{G} \rightarrow \mathrm{R}^{+} \quad$ (Función precio).

5) $\mathrm{U}_{\pi}: \mathrm{J} \times \mathrm{R}^{\mathrm{n}} \rightarrow \mathrm{R} \quad$ para todo $\pi \in \mathrm{J}$ (funciones de utilidad (beneficio)).

Aunque el autor especifica amplia y detalladamente cada concepto, podemos resumir los aspectos fundamentales como sigue ${ }^{6}$.

$J$ es el conjunto de agentes económicos, es decir, aquellos que realizan los intercambios. $G$ es un conjunto finito de clases de bienes; $q$ determina la cantidad de cada clase de bien $\gamma_{1}, \ldots, \gamma_{n}$ del conjunto $G$ en manos de cada persona ( $\pi$ $\in J$ ). Evidentemente la cantidad de bienes no puede ser negativa $\left(\mathrm{R}_{\mathrm{o}}^{+}\right)$. La función precio coordina un número real positivo con cada clase de bien $\gamma$, que es entendido como precio de la clase de bien $\gamma$, exactamente como precio de una unidad $\gamma$. El concepto de utilidad (beneficio) $\mathrm{U}_{\pi}$ es el más complicado. El tuplo $\left\langle\alpha_{1}, \ldots, \alpha_{n}\right\rangle$ designa la cantidades de bienes que la persona $\pi$ podría poseer. Con e la coordina un número real $U_{\pi}\left(\alpha_{1}, \ldots, \alpha_{n}\right)$, que especifica la magnitud de la utilidad que obtiene la persona del consumo o posesión de esta cantidad de bienes.

Un sistema económico considerado a lo largo de un periodo de tiempo supone una distribución de bienes en el estado inicial y otra en el estado final. Para obtener modelos de dos fases que describan el estado inicial y el final hay que

Balzer (1997, p. 90) usa el término función beneficio para referir a la utilidad en un sentido amplio, es decir, a la utilidad del que adquiere un bien y a la del que lo vende en un sistema de intercambio. El dinero es un bien mas del proceso de intercambio portador de utilidad. Nosotros usaremos la noción de función de utilidad ya que es la habitual y nos parece más clara.

${ }^{6}$ Véase W. Balzer, 1997, pp. 87-90. 
incorporar en los modelos dos distribuciones de bienes. Balzer define ambas distribuciones y señala que con los conceptos hasta ahora introducidos se sintetiza una estructura que puede servir como modelo potencial de los sistemas de intercambio que contiene dos fases y la posibilidad de un tránsito qi y qf.

$\mathrm{X}$ es una economía potencial de intercambio, sii existen $\mathrm{J}, \mathrm{G}, p, q i$ y $q f$ y $U$ tales que

l) $<J ; G ; p, q i, q^{f} ; U>$

2) $\mathrm{J}=\left\{\pi_{1}, \ldots, \pi_{\mathrm{n}}\right\} \quad$ es un conjunto finito (de personas).

3) $\mathrm{G}=\left\{\gamma_{1}, \ldots, \gamma_{\mathrm{n}}\right\}=\{1, \ldots, \mathrm{n}\}$ es un conjunto finito (de clases de bienes).

4) $\mathrm{p}: \mathrm{G} \rightarrow \mathrm{R}^{+} \quad$ (Función precio).

5) $q i: \mathrm{J} \times \mathrm{G} \rightarrow \mathrm{R}_{\mathrm{o}}^{+} \quad$ (distribución inicial).

6) $q f . \mathrm{J} \times \mathrm{G} \rightarrow \mathrm{R}_{\mathrm{o}}^{+} \quad$ (distribución final).

7) $\mathrm{U}: \mathrm{J} \times \mathrm{R}^{\mathrm{n}} \rightarrow \mathrm{R}$ es $\mathrm{C} \quad$ (función utilidad (beneficio)) (Balzer 1997: 93).

La clase de todas las economías de intercambio potenciales se designan mediante $\mathrm{Mp}$ (TIE).

La teoría del intercambio describe cómo los agentes tratan de maximizar el valor de la función de utilidad (beneficio) intercambiando la distribución de bienes que poseen . "La teoría nos dice que este proceso está gobernado por ciertas reglas. Si los individuos actúan acorde a esas reglas, la nueva distribución satisfará ciertos requerimientos que pueden ser afirmados en forma de axiomas». (Balzer, 1987:156).

X es una economía de intercambio sii existen J, G, p, qi, qf; y U, tales que

1) <J,G,p,qi, q’ U>

2) $x$ es una economía potencial de intercambio.

3) $q \in \mathrm{LI}_{\mathrm{x}}\left(\mathrm{LI}_{\mathrm{x}}\right.$ es la definición de la limitación de ingresos para $\mathrm{x}$ )

4) para todo $\pi \in J$ y para todo $q$ : si $q \in \mathrm{LI}_{\mathrm{x}}$, entonces $\mathrm{U}_{\pi}\left(\mathrm{q}_{\pi}\left(\gamma_{1}\right), \ldots,\left(\mathrm{q}_{\pi}\right.\right.$ $\left.\left(\gamma_{n}\right)\right) \leq U_{\pi}\left(q_{\pi}^{f}, \ldots, q_{\pi}^{f}\left(\gamma_{n}\right)\right)$ 
5) 5) para todo $\quad \gamma \in G: \sum q^{i}\left(\pi, \gamma_{i}\right)=\sum q^{f}\left(\pi, \gamma_{i}\right)$ (Balzer, 1997:97).

$$
\gamma \in \mathrm{J} \quad \pi \in \mathrm{J}
$$

Los modelos efectivos, $M$ de la teoría, suponen la estructura de los Mp enriquecida con estos nuevos axiomas. La clase de todas las economías de intercambio se designa mediante $\mathrm{M}(\mathrm{TIE})$.

Los axiomas (3), (4) y (5) introducen dos conceptos adicionales limitación de ingreso y valor. Balzer define conjuntistamente estos dos conceptos y a partir de ellos introduce los axiomas que expresan las leyes fundamentales de la teoría. Estos son: 3 ) el axioma que postula que en la distribución final de $q f$ (para todas las personas) el valor de su dotación es el mismo que en su dotación inicial, es decir, el valor total de los bienes que se hallan en su posesión no se han modificado durante el tiempo considerado; 4) el axioma que afirma que la utilidad (beneficio) extraída de la dotación final sea máxima, es decir, la maximización de la utilidad en relación a las posibilidades de intercambio admitidas por la limitación del ingreso. Esta es de hecho la ley fundamental de la teoría (condición necesaria para hallar una distribución de equilibrio); 5) el axioma que asevera que los mercados se despejan (clear) es decir, para cada bien $\gamma_{i}$, la demanda total de $\gamma_{i}$ es igual a la of erta total de $\gamma_{i}$. En un mercado no despejado son diferentes la oferta y la demanda totales.

Destaquemos que en los modelos potenciales $(\mathrm{Mp})$ sólo se postula la existencia de una función de utilidad (beneficio) mientras que en los modelos efectivos (M) de la teoría se exige maximización de dicha función (uno de los dos nuevos axiomas introducidos (4)). Esto supone distinguir dos aspectos, el de la función de utilidad (beneficio) propiamente dicha y el del valor de esa función, la maximizacion de la utilidad (beneficio).

En relación a la t-teoricidad y t-no teoricidad de los términos de la teoría encontramos que la distribución inicial de bienes es un término t-no teórico, viene dada, no se define en la teoría. Los precios son términos t-teóricos ya que la demostración de la existencia de precios de equilibrio requiere del uso sistemático de los supuestos de la teoría. La distribución final es un término t-teórico ya que se determina a través de la teoría. La función de utilidad (beneficio) es tratada como t-no teórico, entendiendo Balzer que presupone una teoría previa: la 
teoria de la utilidad esperada (TUE). Sin embargo, el autor ha dudado sobre este punto y en algún momento la ha tratado como término t-teórico?

Hasta aqứ la axiomatización conjuntista es factible adaptándose a los requisitos habituales. Las dificultades o especificidades aparecen en relación a la teoricidad o no teoricidad de un término básico, la función de de utilidad (relaciones interteóricas); a las constricciones intermodelos (condiciones de ligadura); y al establecimiento del dominio I de aplicaciones (en las que tiene un papel relevante las condiciones de ligadura y las relaciones interteóricas).

\section{La función de utilidad y las relaciones interteóricas.}

Si se entiende la función de utilidad como término no teórico esta función provine de una teoría previa. La mejor candidata, tal como señala Balzer, es la teoría de la utilidad esperada (TUE) propuesta inicialmente por Von Neumann y Morgenstern. Por tanto, la teoría del intercambio mantiene una relación interteórica con la teoría de la utilidad esperada. La relación interteórica se da entre una teoría $T$ y una teoría $T^{\prime}$ 'cuando $T$ proporciona los términos $T^{\prime}$ - no teóri$\cos$ a $\mathrm{T}^{\prime}$. Existe un vínculo interteórico que liga ambas teorías y que se ocupa del trasvase de información de la teoría $T$ a su teorización T'. Esto supone que los términos T'-no teóricos han quedado bien establecidos en $\mathrm{T}$ y que, por consiguiente, existe un método de determinación (medida) para los mismos que no implica a T' (ésta no puede determinar los conceptos). El ejemplo típico viene dado por la afirmación de que la mecánica es una teorización de la cinemática que determina los conceptos de distancia, tiempo, velocidad y aceleración. En nuestro caso la teoría del intercambio es una teorización de la teorfa de la TUE que determina el concepto fundamental de función de utilidad.

Los vínculos interteóricos pertenecen al núcleo de la teoría y, por consiguiente, forman parte de su identidad. No se establecen en el conjunto de axiomas que determinan los modelos efectivos de la teoría dado que esto, por un lado, oscurecería su carácter interteórico y, por otro, supondría una redundancia puesto que aparecerían en los modelos de ambas teorías.

7 W. Balzer, 1982, considera la función de utilidad como téorica. Después de la crítica de Hazlinger modifica su concepción, pasando a entenderla como no teórica en Balzer, 1985, y Balzer, 1997. 
La consideración de la función de utilidad como un término TlEi-no teórico se basa en el supuesto de que el término está bien determinado (medido) en la TUE. Si no se cumple esta condición, la relación interteórica se oscurece y la teoría a la que se da el trasvase de información queda afectada; tiene consecuencias sobre la identidad de la misma y sus aplicaciones intencionales (lo que apunta hacia la cuestión de su contenido empírico). Esto es lo que ocurre en el caso que estamos analizando. La teoría presenta dificultades para constituirse como una preteoría adecuada de TIE al presentar problemas en la determinación (medición) de U. Estos problemas tienen que ver con el incumplimiento de los axiomas que definen la función de utilidad.

La axiomatización de Von Neumann y Morgenstern da cuenta de las condiciones que posibilitan la construcción de una función de utilidad para elecciones bajo riesgo con las mismas herramientas utilizadas en la construcción de una función para elecciones con consecuencias ciertas (existen otras axiomatizaciones de la teoría). Además el axioma de continuidad propuesto por los autores proporciona una técnica que haría posible la medición de la utilidad hasta una transformación lineal, lo cual puede ser entendido como la posibilidad de construir una función en la que la utilidad pudiera ser medida cardinalmente.

Esta posibilidad, sin embargo, ha sido puesta en duda dados los numerosos problemas relacionados con las comparaciones interpersonales de utilidad y la utilidad marginal decreciente. Aspectos en los que se centraron los ataques de Robbins al enfoque cardinalista, que supusieron un fuerte cuestionamiento de la cardinalidad y el predominio del enfoque ordinalista basado en la utilización de funciones de utilidad ordinal. La ortodoxia economía neoclásica mantiene que la medición de la utilidad hasta una transformación lineal no tiene ningún significado en lo que se refiere a inferir estados mentales subjetivos de satisfacción o insatisfacción por parte de los agentes. Esto es igual en el caso de la incertidumbre:

ninguna observación de elecciones en régimen de incertidumbre puede justificar la conclusión de que pueden coinpararse los cambios en el nivel de satisfacción subjetiva de un consumidor. Si la satisfacción puede ser creciente a un ritmo decreciente a medida que aumenta la riqueza, esto es solo la con- 
secuencia de una decisión puramente arbitraria acerca de cómo se ha de definir la utilidad esperada (Green, 1976:228).

En cuanto a los axiomas que definen la función de utilidad, la fundamentación axiomática de la teoría de la utilidad esperada se basa en cuatro axiomas cuyo incumplimiento afecta profundamente a la teoría y la enfrenta a importantes problemas. Estos axiomas son: el de cancelación (también conocido como axioma de sustitución de Neumann y Morgenstern, principio de cosa segura de Savage o condición de independencia de Luce y Krantz), el de dominancia, el de invarianza (formulado como de extensionalidad por Arrow) y, finalmente, el de transitividad. La maximización de la utilidad esperada es una función de estos cuatro supuestos, por tanto, su satisfacción afecta a la ley de maximización de ]a utilidad de la teoría del intercambio. Cancelación, transitividad, dominancia e invarianza caracterizan las ordenaciones de preferencias, son condiciones necesarias para la construcción de una función de utilidad y para poder hallar un valor máximo para dicha función.

Con la cancelación se exige la eliminación de cualquier estado del mundo que produzca el mismo resultado independientemente de la elección del agente. El axioma de transitividad exige la transitividad en la ordenación de preferencias de los agentes. L: dominancia afirma que si una opción es mejor que otra en un estado, y al menos tan buena como la otra en todos los demás, la opción dominante será la elegido. Y la invarianza asevera que diferentes representaciones del mismo problema de elección producirán la misma preferencia.

Lo que han mostrado diversas investigaciones es que estos axiomas se incumplen. Los trabajos de Allais (1953) y de Ellsberg (1961) establecieron que cada uno de estos axiomas es vulnerado por los decisores en situaciones de riesgo e incertidumbre. Igualmente May (1954) muestra, partiendo de resultados experimentales, el incumplimiento de la transitividad. Lichtenstein y Slovic (1971) señalan lo mismo para contextos estocásticos con fenómenos de inversión de pre-

${ }^{8}$ A pesar de las criticas generales a la cardinalidad en el terreno de la teoría de la elección social los trabajos sobre mediciones cardinales de la utilidad han continuado con autores como J. Harsanyi,1955, quien defiende el uso de comparaciones interpersonales de utilidad para construir una función de bienestar social cardinal que sea igual a la media aritmética de las utilidades de todos los individuos de la sociedad, o el enfoque de comparabilidad parcial de A. K. Sen, 1970,1982. 
ferencias (preference reversal). Tversky y Kahneman (1974, 1988, 1990, 2000) prueban el incumplimiento del axioma de la dominancia que constituye un supuesto fundamental de la teoría de Von Neuman y Morgenstern y, también, el axioma de la invarianza lo que muestra la inconstancia de la función de utilidad. La cláusula ceteris paribus que afecta a la constancia de dicha función presenta problemas cuando la cuestión no puede ser explicada como un cambio de gustos en el tiempo sino como una diferencia en la estructuración del problema de elección. Kahnemann y Tversky explican desde su Prospect Theory las desviaciones de la invarianza y de la dominancia por efecto de la composición del problema y de los fallos en el razonamiento y juicio estadísticos. Muestran cómo se incumplen los supuestos fundamentales de la TUE y el teorema fundamental de la maximización que afirma que los agentes elegirán aquellas opciones cuya utilidad esperada sea más alta. La TUE falla en $r$ acoger lo que son las actitudes de la gente hacia el riesgo y la forma en que procesan la información cuando hay incertidumbre acerca de los resultados $y$, por consiguiente, se mueven con probabilidades. La Prospect Theory pone en cuestión la afirmación central de que la forma de la función de utilidad será cóncava. Teniendo en cuenta que Balzer propone la concavidad de la función de utilidad como una especialización teórica de TIE, y que el contenido empírico de las teorías viene dado en buena medida por las leyes especiales (especializaciones teóricas), esto tiene efectos sobre el status empírico de TIE?

Las consecuencias del incumplimiento de los axiomas señalados han sido interpretadas de maneras diferentes. Se ha entendido que ello no afecta a la TUE de forma sustancial, ya que lo que ésta ofrece es un tratamiento idealizado respecto al cual han de ser valorados los casos de incumplimiento, sin por ello cuestionar la teoría. Pero también se ha considerado que los casos de incumplimiento exigen teorías específicas complementarias de la TUE descriptivas, no normativas, y, finalmente, que este hecho pone en cuestión la viabilidad de la TUE.

Lo que está claro es que la teoría de la utilidad esperada tiene un status problemático y esta problematicidad afecta a TIE en aspectos fundamentales como es la determinación de un concepto básico, tiene consecuencias sobre una de sus

\footnotetext{
${ }^{9}$ W. Balzer, 1979, p. 1 05. Para este tema véase Obdulia Torres, Tesis Doctoral p. 292.
} 
leyes centrales y sobre las aplicaciones de la teoría. En relación a las aplicaciones de la teoría la idea básica es que:

cuando $T_{1}$ se considera teorización de $T_{0}$ es porque toda aplicación intencional $\mathrm{x}$ de $\mathrm{T}_{1}$ (es decir, toda estructura que representa un upedazo de realidad" al que se pretende aplicar $T_{1}$ ) tiene una subestructura y udeterminada por $T_{0}$ " en el sentido de que cumple sus leyes, esto es, $y$ es un inodelo actual de $T_{0}$ (o parte de un modelo actual de $T_{0}$ (Díez, Moulines, 1997:372).

En este sentido los axiomas señalados y el supuesto de la maximización de la TUE determinarían la subestructura de cada aplicación. Pero en la medida en que los axiomas presentan problemas de incumplimiento, las aplicaciones intencionales de TIE quedan afectadas al menos en el sentido de que los «trozos de realidad" a los que se aplica son aquellos que presentan la estructura determinada por TUE; pero estos se limitan a contextos informativamente transparentes, como los que se dan en el laboratorio, en los que se cumplen los axiomas de la $\mathrm{TUE}^{10}$. Balzer expresa su escepticismo respecto a la aplicación de TIE a sistemas reales, puesto que la determinación (medición) de la función de utilidad sólo funciona en situaciones de laboratorio. No lo hace en las situaciones de intercambio reales, lo que produce una situación poco satisfactoria y " explica por qué la teoría "no suministra ni puede suministrar pronósticos precisos» (Balzer, 1997: 125).

En cuanto a la necesidad de que exista alguna teoría subyacente para determinar las utilidades, y la TUE parece ser la mejor candidata, Balzer afirma que TIE debe tener alguna teoría subyacente de las preferencias de cara a determinar sus utilidades. Sin embargo, dado que la naturaleza de la teoría subyacente no está clara. dej aromos la forma específica de este vínculo como una cuestión abierta. (Balzer, 1987:161). Posteriormente es más radical. Después de analizar la teoría de la utilidad esperada mantiene que si existiese una preteoría de TIE, establecida, que clarificase el significado de la utilidad o de la referencia de un modo satisfactorio, TIE se podría establecer sobre ella. Pero desgraciadamente esto no es asi ${ }^{11}$. (Balzer,1997:125).

10 Hay dificultad para establecer las aplicaciones intencionales de la teoría ya sea por el método de autodeterminación ya por el paradigmático.

11 Balzer usa la abreviatura ECO. En la traducción usamos la abreviatura española TIE. 
Una forma de evitar los problemas asociados a la TUE es considerar la función de utilidad como un término TIE-teórico ( primera opción escogida por Balzer 1982). En este caso los axiomas que caracterizan a la ordenación de preferencias, reflexividad, transitividad continuidad y completad formarían parte de la teoría ya que son condiciones necesarias para la construcción de una función de utilidad y para poder hallar un valor máximo para dicha función. Por tanto, los problemas señalados se trasladan al seno de TIE.

Si se entiende la función de utilidad como TIE-teórica, la situación señalada queda reflejada en la problemática de las aplicaciones de la teoría para predecir precios y cantidades de mercancías:

tan pronto como uno acepta la TIE- teoricidad de la utilidad (U )esta forzado a rendirse. El argumento funciona así. La derivación de precios o cantidades desde utilidades dadas por medio de TIE puede producir proposiciones empíricas o predicciones sólo si TIE es aplicado a un sistema concreto y si es posible comprobar que este sistema es un modelo de TIE. Pero, para comprobar si algún sistema es un modelo de TIE es necesario determ inar los valores de las funciones que ocurren en TIE, especialmente de U. La determinación de los valores de U presupone - por el criterio de teoricidad- que el proceso de determinación ya es un modelo de TE. Si uno quiere comprobar si este proceso de determinación en efecto es un modelo de TIE uno está en la misma situación que al principio (Balzer, 1982:36).

La cuestión es, como reconoce el autor, que el significado de la función de utilidad no se establece mediante la teoría de intercambio y además se carece de una teoría que clarifique su significado ${ }^{12}$. TIE aparece, pues, como una teoría en la que hay dificultades para determinar uno de sus conceptos básicos. Esto tiene consecuencias sobre la que para muchos es su ley fundamental, la de la maximización de la utilidad, y sobre las aplicaciones de la teoría, como se hace patente en relación a las condiciones de ligadura que quedan afectadas por la problemática de las funciones de utilidad.

12 W. Balzer, 1997, pp. 124-25. 


\section{Las condiciones de ligadura}

Las condiciones de ligadura afectan a las relaciones intermodelos. Se aplican a los valores de las funciones de los distintos modelos; estos han de ser los mismos en cada modelo en el que aparezca la misma función. Esta restricción afecta a las aplicaciones de la teoría (dominio I), ya que el valor de una función en una aplicación de la teoría no puede ser distinto de los valores en otra aplicación (si las diversas aplicaciones de una teoría como es de esperar no son modelos disjuntos). El valor de la función masa para la Tierra es invariante independientemente de que el modelo considerado sea Tierra-Luna, Tierra-Sol, Tierra- proyectil, etc. La estructura de esta constricción se puede representar como una relación entre los dominios de las funciones teóricas y sus rangos de tal manera que si entre los elementos de la unión de dominios de las funciones vale la relación $\mathrm{B}$, entonces entre los elementos de los rangos de esas funciones vale la relación B'. Si representamos la Tierra por $a$ en el primer sistema visto y al en el segundo, de forma que tenemos las funciones:

\section{a ? \\ al_?}

Ia relación entre los dominios de las funciones teóricas es la de igualdad:

$$
\text { si } \mathrm{a}=\mathrm{al} \text { entonces } \mathrm{n}(\mathrm{a})=\mathrm{n}(\mathrm{al}) \text {. }
$$

Los modelos de la teoría aparecen así ligados por esta condición. Esto permite, una vez establecido el valor de una función, darlo por supuesto en los distintos modelos de la teoría, es decir, se da por sentado que allí donde aparezca dicha función el valor establecido se cumplirá dado que la función es constante. Lo que significa que se pueden hacer pronósticos transportando la información a otros modelos y aplicaciones. La capacidad predictiva (y explicativa, teoría depende de ello.

En la reconstrucción de Balzer (1977:134-35) el valor de la función de utilidad ha de permanecer constante para los agentes económicos a través de los diversos sistemas económicos de intercambio de tal manera que, establecido en un sistema sus valores o proporciones para determinadas cantidades de bienes se podrían presuponer en el siguiente sistema y con su ayuda averiguar valores de utilidad para nuevas cantidades de bienes. 
La constancia de la función supone que los modelos de la teoría no están aislados entre sí y que, establecido el valor de la función, puede presuponerse en otro modelo Esto permitiría «la trasposición de datos, que se conocen o se han calculado para una determinada situación, a otra situación»(Balzer 1997:134). Lo que supone que esa otra situación está vinculada con la originaria de diferentes maneras.

Balzer reconoce que esto difícilmente ocurre, ya que la constancia de la función utilidad sólo se sostiene en términos ceteris paribus. Es decir, en el tránsito de un sistema económico a otro la función de utilidad se trata como si no cambiase. Para poder hacer esto se considera que los individuos son constantes en sus propiedades relevantes, lo que significa constancia en preferencias, carácter, gustos y hábitos de consumo. Pero tal constancia no se da, estas propiedades cambian frecuentemente y además es difícil trazar una frontera entre "propiedades económicamente relevantes de una persona y las Influencias económicamente relevantes a las que está expuesta, y aquellas Influencias y propiedades que no son relevantes económicamente» (Balzer, 1997:133-135). Estas dificultades son las que se evitan con el uso de cláusulas ceteris paribus. La problemática analizada más arriba muestra que la inversión de preferencias y, especialmente, el incumplimiento del axioma de la invarianza constituyen ejemplos claros de cambios en los órdenes de preferencia.

El problema de la constancia de la función de utilidad significa que los modelos de la teoría del intercambio no están ligados en el sentido en que lo están los de la mecánica clásica de partículas y no se puede extrapolar la información. Una vez calculados los valores de utilidad para un sistema no podemos presuponerlos en otro y establecerlos para nuevas cantidades de bienes. Esto hace que la teoría pierda potencia explicativa y predictiva.

En la reconstrucción de la teoría del valor neoclásica el problema aparece de nuevo en relación a la función de utilidad. Lo que se intenta es encontrar una función valor que cumpla las condiciones de ligadura para los distintos modelos de la teoría. Esta función valor se basaría en la función de utilidad que se pretende métrica y transmodélica. La condición de ligadura supone que el valor de utilidad de una mercancía para un consumidor en un sistema de intercambio tiene que ser igual al valor de utilidad de la mercancía para el consumidor en otro sistema. Es decir, que la función utilidad es constante. Pero esto no es así por las 
razones antes aducidas. La constancia en los gustos, por ejemplo, se podría sostener si los sistemas de intercambio fuesen completamente equivalentes, pero, como señala Alex Costa (1987), éste, es un subconjunto irrelevante de los sistemas de intercambio. Se carece, por tanto, de una Función que permita predecir los movimientos de los precios como la función masa permite la predicción de las posiciones de los cuerpos. No hay expansión teórica para los fenómenos de intercambio de mercancías con una función de utilidad válida en más de un sistema concreto que conecte los valores de utilidad con los de los precios. La teoría del valor neoclásica no dispone de la condición de ligadura necesaria para dar cuenta de las variaciones de los precios.

De esta manera, se puede dar cuenta de los precios en sistemas ideales de intercambio pero no de los precios reales, y ésta parece ser la vía que siguen los economistas neoclásicos. Lo que supone, como afirma J. Mosterín, que las teorías económicas describen ciertas estructuras con más o menos precisión, pero no está claro que ninguna de ellas tenga modelos reales:

Así determinadas teorías económicas sólo serían aplicables (sólo tendrían coino inodelos) a sistemas económicos donde la competencia, transparencia y elasticidad de ciertos factores fueran perfectas. Mientras no exista ninguna economía de esas característica., dichas teorías carecerán de modelos reales (Mosterín, 1984:150).

Tal idea es aceptada por el mismo Balzer, quien reconoce que no se ha podido afirmar de forma general y sistemática a qué sociedades se aplican los axiomas de la teoría de intercambio ${ }^{13}$. Esto evidencia el problema del componente empírico de las teorías de la economía neoclásica que ha interesado a los filósofos y economistas desde el S. XIX.

\section{El problema del componente empírico}

Lo que ha permitido precisar el análisis estructural de la teoría del intercambio (igual en el caso de la teoría del valor) es que el problema del componente empírico tiene que ver con la naturaleza de las relaciones interteóricas y las condiciones de ligadura de la teoría, y con cómo ello afecta a las aplicaciones efectivas I

${ }^{13}$ W. Balzer, 1997, p. 126. 
de la teoría. Lo que está relacionado con las dificultades de determinación (medición) de un concepto fundamental de la teoría como es el de función de utilidad y el establecimiento de un valor máximo para dicha función (ley de la maximización de la utilidad).

Todo ello muestra la importancia que para la teoría del intercambio tiene la investigación y la elaboración de propuestas que puedan paliar los problemas que plantea la determinación de las funciones de utilidad. Esto ha sido abordado por autores como $\mathrm{H}$. Simon, y los mencionados en relación a la crítica de la TUE, al intentar elaborar una teoría menos idealizada con base en la investigación empírica y la experimentación del comportamiento de elección de los agentes. Ellos coinciden con otros especialistas en señalar como un serio problema de las teorías de la economía el deslizamiento que se ha dado hacia el normativismo, formalismo y el interés por el desarrollo matemático con escasa preocupación empírica. Esto ha hecho que se ponga en cuestión la naturaleza de estas teorías: se basan en supuestos y leyes formales muy idealizadas con un insuficiente rendimiento empírico. De esta manera, principios fundamentales, como el la maximización de la utilidad, han sido entendidos como principios guía de la investigación más que como una ley empírica, usu valor es, usando el término de Kuhn, el de una promesa, el de un poderos principio motriz para hacer ciencia" que no se contrasta con la realidad (Mofllines, 1982:106). El mismo Balzer entiende que la teoría del intercambio, en cualquiera de sus versiones, ha de ser vista como una teoría pura más que estrictamente empírica.

Cuando se estudia la historia de la economía de intercambio y, más genéricamente de la teoría de la utilidad, rara vez se encuentran aplicaciones en sistemas económicos concretos. En lugar de ello, la mayoría de las veces, se demuestran teoremas interesantes. Las argumentaciones en favor o en contra de la corrección de la teoría proceden rara vez de investigaciones empíricas, de datos reunidos o medidos, sino que se refieren a la generalidad, plausibilidad y elegancia de tales teoremas. Parece por ello oportuno emplear también junto al concepto de teoría empírica el concepto de teoría pura (Balzer, $1997: 133)^{14}$.

14 En esta línea E. Händler plantea la reconstrucción de la teoría microeconómica del equilibro como una teoría pura. "De este modo, un elemento teórico puro es una \$-tupla ( $\mathrm{Mp}, \mathrm{M}, \mathrm{C}$, A), los primeros tres componentes representan los elementos de la estructura, siendo A el conjunto 
Diferentes voces críticas, desde los empiristas de principios del siglo XX hasta autores recientes de diversas adscripciones, coinciden con este diagnóstico, indican la necesidad de hacer de la economía una ciencia con mayor contenido empírico, señalan cuáles son las causas que dificultan el logro de este objetivo y proponen algunas vías para lograrlo. Así, realistas como Lawson $(1989,1994)$ han cuestionado la práctica de formular axiomas de amplia generalidad, aunque de poca capacidad explicativa, típica de la economía neoclásica. Los axiomas no dan cuenta de la naturaleza de los mecanismos y estructuras económicas que rigen los hechos económicos observables. Axiomas como los relacionados con la utilidad o la competición perfecta son idealizaciones que no tienen que ver con el modo en que operan los mecanismos económicos reales, son introducidos para lograr tratabilidad matemática. En un sentido parecido encontramos las críticas de Mäki (1992) a la economía neoclásica: se supone que los consumidores son individuos totalmente informados, calculadores racionales y maximizadores de beneficio, lo cual no se ajusta a la realidad. Las mismas empresas, como han indicado Hall y Hitch (1939), no son maximizadoras en el sentido de que no se llevan a cabo los cálculos y ajustes marginalistas requeridos. Las hipótesis acerca de las empresas o los consumidores dejan fuera otras motivaciones (altruismo, motivación personal, etc). Las consecuencias de todo esto son, para Mäki, que la economía neoclásica no da una descripción teórica adecuada ni de las empresas reales ni de los consumidores y su contenido empírico es escaso. Ambos autores destacan la importancia del surgimiento de alternativas como la de Kaldor (1985), quien propuso una teoría del equilibrio alternativa a la ortodoxa. Su propuesta de un concepto de tiempo irreversible, real e histórico presta más atención al contexto y las circunstancias. Robinson (1977) señaló que era imposible asumir la constancia de nada en el tiempo (por ejemplo de las preferencias). Es necesaria una descripción más afinada del modo en que opera el mercado, del comportamiento de los agentes y de la formación de precios, una definición más precisa de sus estructuras y operaciones.

Igualmente desde posiciones empiristas recientes se considera que el fuerte desarrollo de las matemáticas en economía y la fascinación por el trabajo neowalrasiano han supuesto una perspectiva cada vez más restringida en lugar de aumen-

de aplicaciones de esta estructura: A es un conjunto de sistemas económicos descrito por ejemplos paradigmáticos en la literatura estándar que no refieren a mundos posibles realmente existentes" (Händler, 1980, p. 51). 
tar la perspicacia científica para dar cuenta de la complejidad de los avanzados sistemas económicos. Los teóricos de la economía se cierran cada vez más sobre la teoría ${ }^{15}$.

\section{Conclusión}

Para concluir, puede señalarse que la axiomatización conjuntista de teorías sociales como la examinada, aunque peca de cierta artificiosidad, se muestra como un potente instrumento para clarificar diversos aspectos tanto del componente conceptual como del empírico de las teorías, mostrando el estado de las mismas, los puntos que hay que investigar y los que están sólidamente establecidos. La reconstrucción da como resultado una clarificación y precisión de las especificidades y problemas que presentan las teorías sociales difíciles de alcanzar por otros medios, siendo crucial para la discusión de cuestiones teóricas y epistemológicas relacionadas con ellas ${ }^{16}$.

\section{Bibliografía}

ALLAIS, P. M., 1953, «The behavior of rational man in risk situations. A critique of the axioms and postulates of Ameritan School", Econometrica, 21, 503-546.

Allingham, M. 2002: Choice Theory: A Very Short Introduction, Cambridge, Cambridge University Press.

BALZER, W., 1982, «A Logical Reconstruction of Pare Exchange Economics», Erkenntnis, 17, 23-46.

BAlZER, W., 1985, "The Proper Reconstruction of Exchange Economics", Erkenntnis $23,185-200$.

Balzer, W., Bert, H., 1989, Philosophy of Economics, Kluwer Academic Publishers (reedición).

BALZER, W.,1997, Teorias empiricas: modelos, estructuras y ejemplos, Madrid, Alianza.

Balzer, W.; Moulines, U. \& SNeEd, 1987, An Architectonics for Science, Dordrecht, Reidel.

15 Como señala T. A. Boylan and P. F. O, Gorman, 1995, p. 201.

16 La redacción de este trabajo ha sido facilitada por la concesión ${ }^{* * * *}$, 
BALZER, W.; MOULINes, U., 1996, Structuralist Theory of Science. Focal Issues, New Results, Walter de Gruyter Inc.

Balzer, W.; Moulines, U. \& SNEED, J. (Eds), 2000, Structuralist Knowlwdge Representation.

- Paradigmatic Examples (Poznan Studies in the Philosophy of the Sciences and the Humanities 75), Amsterdam, Rodopi.

BILLOT, A. B. 1992, Economic theory offuzzy equilibria: an axiomatic analysis, Berlín Springer-Verlag.

Boubarki, N., 1968, Theory of sets, París, Hermann.

BOYLAN, T. A. and O. GORMAN, P. F., 1995, Beyond rhetoric of realism in ec onomics. Towards a reformulation of economic methodology, London, New York, Routledge.

CosTA, A., 1987, "Fundamentos teóricos de economía crítica", Actas de las Jornadas de Economia Critica de Madrid, 232-239.

Debreu, G., 1972, Theory of Value: An Axiomatic Analysis of Economic Equilibrium, Yale University.

DiederiCh, W. y Fiedrich, F. H., 1981, Estructuras Sneedianas en El Capital de Marx, México, UNAM.

Diez, J. A. \& Moulines, U.,1997, Fundamentos de Filosofia de la Ciencia, Barcelona, Ariel.

Dies, J. A. \& Lorenzano, P. (Eds), 2002, Desarrollos actuales de la metatoria estructuralista: problemas y discusiones, Universitat Rovira i Virgili, Universidad Autónoma de Zacatecas.

ECHEVERRIA, J., 1995, Filosofia de la ciencia, Madrid, Akal.

ELLSSERG, D., 1961, “Risk, ambiguity, and the Savage axioms», Quaterly Journal of Economics, 75, 643-669.

GARCIA DE LA SIENRA, A., 1980, «Elementos para una reconstrucción lógica de la teoría del valor de Marx», Crítica, 35, 71-96.

- 1988, "Axiomatic Foundetions of the Marxian Theory of Value». Erkenntnis 29 (1988), 299-341.

- 1992, The logicalioundotions of the Marxian theory of value, Dordrecht, Kluwer Academic.

- 1994, "Idealization and Empirical Adequacy in Economic Theory». En Bert Hamminga y Nino B. De Marchi (eds.). Idealization VI: Idealization in Economics. (Poz- 
nal'n Studies in the Philosophy of the Social Sciences and the Humanities 38). Amsterdam: Rodopi, pp. 117-133.

GreEN, H. A. J., 1976, La teoria del consumidor, Madrid, Alianza Universidad, 1986.

HALL, R. L. and Hitch, C. J., 1939, "Price theory and business behavior», Oxford Economic Papers, 2, 12-45.

HÄNDLER, E., 1980, «The Logical Structure of Moden Neoclassical Static Microeconomic Equilibriun Theory", Erkenntnis 15, 33-53.

Haslinger, F. A., 1983, "Logical Reconstruction of Pare Exchange Economics: An Alternative Viewn, Erkenntnis, vol. 20, 115-129.

HaRSANYI, J. C., 1955, «El Bienestar Cardinal, la Ética Individualista y las comparaciones interpersonales de utilidad", en K. Arrow y T. Scitovsky (comp), Ensayos sobre economía del bienestar, México, F.C.E. 1974.

KAHNEMAN, D. \& TVERSKY, A., 1979, "Prospect theory: An analysis of decision under risk", Econometria, 47: 263-291.

Kahneman, D. \& TVERSKey, A., 1982, "The Psychology of preferences, Scientific American 246: 160-173.

Kahneman, D. \& Tverskey, A., (Eds.) (2000): Choices, Values, and Frames, Cambridge, Cambridge University Press.

Kaldor, N., 1985, Economics without Equilibrium. Cardiff, University College Cardiff Press.

LAwsON, T., 1994, «A Realist Theory for Economics», en R. Backhouse (Ed), (1994) New Directions in Economic Methodology, London, Routledge.

LAwsON, T., 1989, «Abstraction, tendencias and stylised facts: a realist approach to economic analysis", Cambridge Journal of Economics, 13, 59-78.

LiCHTENSTEIN, S. and Slovic, P., 1971, "Reversal of Preferences Between Bilds and Choices in Gambling Decisions", Journal of Experimental Psichology, 89.

MAKI, U., 1992, "Friedman and realism», Research in the History of Economic Thought and Metholodoly, 10, 1-36.

Mas-Colell, et al., 1995, Microeconomic Theory, Nueva York, Oxford University Press.

MAY, K. O., 1954, "Intransitivity, Utility and the Aggregation of Preference Patterns", Econometrica, Vol. 22, 1-13.

MCKInSEY, J. C.; SUgAR, A. C. \& SuPpes, P., 1953, “Axiomatics Foundations of Classical Particles Mechanics», Journal of Rational Mechanics and Analysis, 2, 253-72. 
Mosterin, J., 1984, Conceptos y teorias en la ciencia, Madrid, Alianza.

MOULINES, U., 1982, Exploraciones metacientificas. Estructura, desarrollo y contenido de la ciencia, Madrid, Alianza.

RoBINSON, J., 1977, "What are the questions?», Journal of Economic Literature, 15, (44), 18-39.

SEN, A., 1970, Elección Colectiva y Bienestar Social, Madrid, Alianza Universidad, 1976.

SEN, A. and Williams, B., 1982, Utilitarianissm and Beyond. Cambridge, Cambridge University Press.

SNEED, J., 1979, The Logical Structure of Mathematical Physics, Dordrecht, Reidel.

Selten, R., 1994, "An Axiomatic Approach to Consumer "Welfare" , Mathematical ocial Sciences, 27, 1, 19-30.

STEGMÜLleR, W., 1975, Estructura y dinámica de teorías, Barcelona, Ariel, 1983.

STEGMÜLLER, W., 1979, La concepción estructuralista de las teorias, Madrid, Alianza, 1981.

STIGUM, B. P., 1990, Toward a formal science of economics: the axiomatic method in economics and econometrics, Cambridge, MIT Press.

SUPPES, P., 1989, The Semantic Conception of Theories and Scientific Realism, Illinois, University of Illinois, Press.

TORRES, O., 2002, El problema de las reglas de elección social en la teoría de la acción colectiva, Servicio de Publicaciones de la Universidad de la Laguna.

- «El contenido empírico de la teoría microeconomía según la concepción estructural", Teorema, en prensa.

TVERSKY, A. \& KAHNEMAN, D., (1974): "Judgement under incertainty: Heuristics and biases», Science, 185:11244-1130.

- (1988): "Contingent weighting in judgment and choice", Psychological Review, 95:371384.

- (1990): «The causes of preference reversal», American Economic Review, vol. 80,1:204217. 